\title{
TRANSPORT OF MICROSPHERES BY THE HUMAN OVIDUCT IN VITRO AND THE EFFECT OF TREATMENT WITH MEGESTROL ACETATE
}

\author{
S. AVENDAÑO,* J. PELAEZ* and H. B. GROXATTO $\dagger$ \\ * Ctr. de Investigaciones en Reproducción, Hospital Barros Luco-Trudeau, Universidad \\ de Chile, and $\dagger$ Laboratorio de Endocrinología, Instituto de Ciencias Biologicas, \\ Universidad Católica de Chile, Santiago, Chile
}

(Received 19th Fanuary 1971)

There is little fundamental knowledge concerning the safe and timely transport of the developing egg into the uterus in the human. The present investigation was designed to test the feasibility of studying in vitro the transport of particulate matter by the human oviduct. In addition, since some of the oviducts were obtained from patients treated with megestrol acetate (MA $=17$-alphaacetoxy-6-methylpregna-4,6-diene-3,20-dione), a comparison was made of the transport in the tubes of these patients and in those obtained from a control group.

Twenty-two oviducts were obtained from healthy fertile women at the time of laparotomy for surgical sterilization. Nine of these were obtained from women who had been taking $0.5 \mathrm{mg}$ MA daily for several months up to the time of the operation. The remaining thirteen were from women who had not used any contraceptive for at least 1 month before the operation. The majority of the cases (seven out of nine and ten out of thirteen, respectively) were in the progestational phase of the cycle at the time of surgery, as shown by the presence of young corpora lutea in one of the ovaries. All the oviducts were excised by cutting the junction of the isthmus and the interstitial portion. Immediately afterwards, the tubes were placed in sterile Tyrode solution and taken to the laboratory. In order to study their transport capacity, they were placed in an incubation chamber which consisted of a set of two shallow vessels held $1 \mathrm{~cm}$ apart and contained inside a Wheaton staining dish. One of the shallow vessels, designated the ovarian vessel, contained a small magnet and $4 \mathrm{ml}$ of a suspension of Sephadex G-100 beads (Pharmacia, Sweden) in Tyrode solution $(3.5 \mathrm{mg} / \mathrm{ml})$. The other vessel, designated the uterine vessel, was filled with the same volume of Tyrode solution but without Sephadex beads. All the materials used had been sterilized previously in the autoclave. The infundibular end of the tube was placed in the ovarian vessel and the isthmic end in the uterine vessel. The incubation chamber was sealed and placed over a magnetic stirrer for $1 \mathrm{hr}$ to ensure that the microspheres remained in suspension, thus facilitating uptake by the fimbria. After this, the chamber was transferred to an incubator and left for $72 \mathrm{hr}$ at $30^{\circ} \mathrm{C}$. At the end of this period, a small segment of the fimbria was observed under the microscope to determine the presence of 
motile cilia. These were found in all cases and this index was used as an indication that the tissue was viable. No attempt was made to assess either the sterility of the incubation media or the muscular activity of the oviduct at the end of the period of incubation. Each oviduct was cut at 2.5 to $3 \mathrm{~cm}$ from each end to obtain three pieces corresponding roughly to the infundibulum, the ampulla and the isthmus. They were flushed individually with Tyrode solution to determine the presence, the number and the size of the spheres.

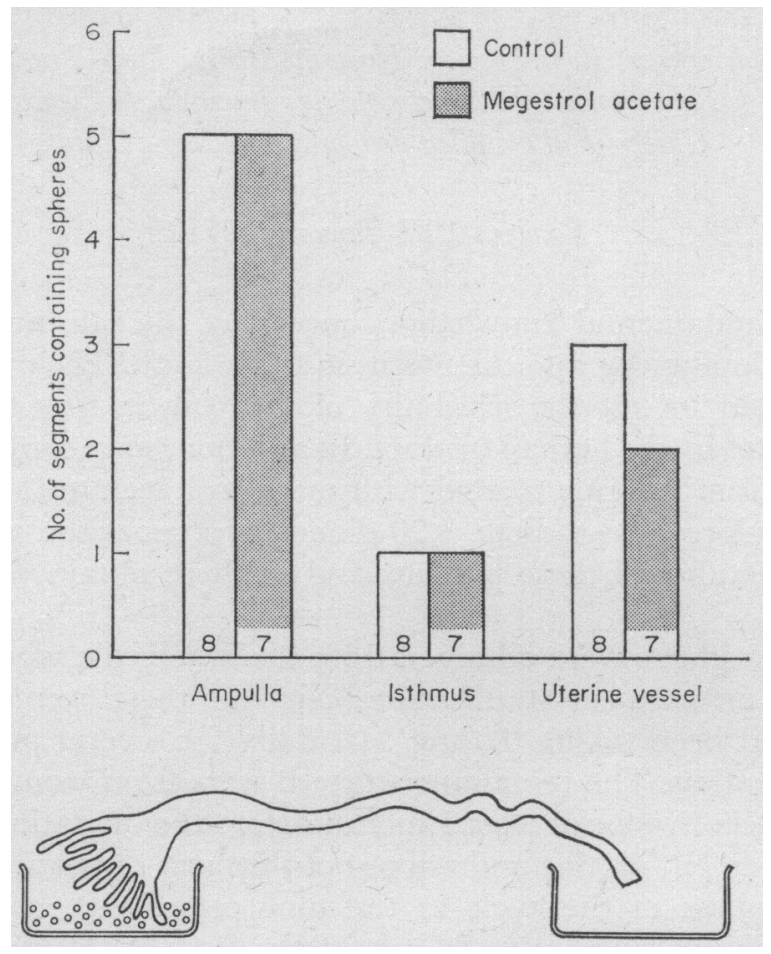

TEXT-FIG. 1. Location of microspheres transported by human oviducts in vitro. The figures at the bottom of columns indicate the number of segments examined.

Because of the obvious contamination of the infundibular segment with the suspension of spheres, the data obtained from this portion of the tube were not included. It was assumed that transport had occurred when spheres were found in the ampulla, the isthmus, the uterine vessel or in two or three of them. Transport occurred in eight of the controls and in seven of the group treated with MA. The mean number of spheres transported was seven and forty-nine, the maximum numbers transported by a single oviduct being 100 and 243 in each group, respectively. The frequency with which each segment was found to contain spheres is shown in Text-fig. 1. There were no differences between groups but there was a clear difference between segments. Thus, in both groups, the ampulla was the segment that contained spheres more frequently and the isthmus was the segment that most frequently did not contain spheres. The data from both groups were combined and analysed by Fisher's exact probability test. This showed that the number of ampullae which contained 
spheres differed significantly from the number of isthmic segments which contained spheres $(P=0.013)$. The number of spheres found in each segment is shown in Table 1.

There was considerable variation between oviducts in the number of spheres transported. In spite of this, their distribution between the isthmus and the two other segments was markedly and consistently different. Most of the spheres were in the ampulla or had passed into the uterine vessel and less than $1 \%$ was found in the isthmus. Furthermore, in the controls, the larger percentage of spheres was in the ampulla whereas in the group treated with MA, the larger percentage was in the uterine vessel. The analysis of these data by the Chi square test showed that the distribution of spheres in the controls differed significantly from that of the treated group $(P<0.001)$. Notwithstanding,

TABLE 1

TRANSPORT OF MICROSPHERES BY THE HUMAN OVIDUCT in vitro

\begin{tabular}{|c|c|c|c|c|c|c|c|}
\hline \multicolumn{4}{|c|}{ Control } & \multicolumn{4}{|c|}{ Megestrol acetate } \\
\hline Oviduct & Ampulla & Isthmus & $\begin{array}{c}\text { Uterine } \\
\text { vessel }\end{array}$ & Oviduct & Ampulla & Isthmus & $\begin{array}{c}\text { Uterine } \\
\text { vessel }\end{array}$ \\
\hline A & 1 & 0 & 0 & I & 0 & 1 & 0 \\
\hline B & 0 & 0 & 1 & $\bar{J}$ & 1 & 0 & 0 \\
\hline C & 52 & 0 & 0 & $\mathbf{K}$ & 6 & 0 & 0 \\
\hline D & 0 & 1 & 0 & L & 3 & 0 & 0 \\
\hline $\mathrm{E}$ & 3 & 0 & 0 & $\mathbf{M}$ & 100 & 0 & 19 \\
\hline $\mathbf{F}$ & 100 & 0 & 2 & $\mathbf{N}$ & 0 & 0 & 243 \\
\hline G & 0 & 0 & 60 & 0 & 70 & 0 & 0 \\
\hline $\mathbf{H}$ & 1 & 0 & 0 & & & & \\
\hline $\begin{array}{c}\text { Total } \\
\%\end{array}$ & $\begin{array}{r}157 \\
71\end{array}$ & $\begin{array}{l}1 \\
0.5\end{array}$ & $\begin{array}{l}63 \\
28 \cdot 5\end{array}$ & & $\begin{array}{l}180 \\
40 \cdot 6\end{array}$ & $\begin{array}{l}1 \\
0 \cdot 2\end{array}$ & $\begin{array}{c}262 \\
59 \cdot 2\end{array}$ \\
\hline
\end{tabular}

* Number of spheres found in each segment at $72 \mathrm{hr}$.

Table 1 shows clearly that this difference was due to a single tube (N) which transported 243 spheres to the uterine vessel. Therefore, the present data do not seem to warrant the conclusion that the oviducts from patients treated with MA consistently transport more spheres into the uterine vessel than the controls.

The spheres from the ovarian vessel and from the uterine vessel were photographed at the same magnification and their diameters were measured from enlarged prints. The mean diameters obtained were $132 \mu \mathrm{m}$ and $137 \mu \mathrm{m}$, respectively. Thus, there was no differential transport of small and large spheres within the size limits of Sephadex G-100 in Tyrode solution (76 to $198 \mu \mathrm{m}$ diameter). Transported spheres were often found partially or completely surrounded by cells adherent to their surface, and in many instances they were also embedded in mucus.

The results indicate that the human oviduct in vitro is capable of transporting synthetic spheres of a size similar to that of denuded ova, from the infundibulum to the isthmus. Treatment in vivo with microdoses of MA does not effect a consistent change in the pattern of transport in vitro. A salient feature of the 
transport was the uneven distribution of the spheres with a marked accumulation in the ampulla and beyond the isthmus. This pattern is in keeping with the concept that in the human, as in other mammals, the isthmic-ampullary junction plays a significant rôle in the regulation of egg transport to the uterus (Greenwald, 1961).

This work was supported by grants from the Population Council to S.A. and H.B.G., which are gratefully acknowledged.

\section{REFERENCE}

Greenwald, G. S. (1961) A study of the transport of ova through the rabbit oviduct. Fert. Steril. 12,80 . 